\title{
Modification of multiple endocrine neoplasia 2A phenotype by cell membrane proximity of RET mutations in exon 10
}

\author{
Andreas Machens ${ }^{1}$, Steffen Hauptmann ${ }^{2}$ and Henning Dralle ${ }^{1}$
}

Departments of ${ }^{1}$ General, Visceral and Vascular Surgery and ${ }^{2}$ Pathology, Martin Luther University Halle-Wittenberg, Ernst-Grube-Straße 40, D-06097 Halle (Saale), Germany

(Correspondence should be addressed to A Machens; Email: andreasmachens@aol.com)

\begin{abstract}
Rearranged during transfection (RET) germ-line mutations in exon 10 are peculiar because they produce both gain-of-function multiple endocrine neoplasia $2 \mathrm{~A}$ and loss-of-function Hirschsprung's disease phenotypes. Drawing on 38 medullary thyroid cancer patients harboring germline mutations in codon $620(n=8), 618(n=19), 611(n=10)$, and $609(n=1)$, this study aimed to test the hypothesis that closer proximity of RET germ-line mutations in exon 10 to the cell membrane may translate into earlier or more advanced disease. The closer mutations in codon 620,618 , and 611 were located to the transmembrane domain (codons 657-636) of the RET receptor, the greater were mean primary tumor diameters $(23.5,18.7$, and $7.5 \mathrm{~mm}, P=0.020)$, the frequency of lymph node metastasis $(75,68$, and $30 \%, P=0.11)$ and pheochromocytoma $(38,16$, and $0 \%, P=0.11$ ). Periods of observation were broadly comparable for these groups (mean age 33.4-39.3 years; $P=0.71$ ). When mutations in adjoining codons were collapsed (codons 620/618 vs $611 / 609)$, the differences in mean primary tumor diameter $(20.1 \mathrm{vs} 7.4 \mathrm{~mm}, P=0.005)$ and lymph node metastasis ( 70 vs $36 \% ; P=0.07$ ) were accentuated. Compared with 80 carriers of exon 11 mutations (codon 634, $n=78$; codon 630, $n=2$ ), the 38 carriers of exon 10 mutations, which are rarer and confer a weaker transforming activity in vitro than exon 11 mutations, required significantly more time to develop fewer tumors. Although limited in numbers, these data suggested that membrane proximity is an important determinant of tumor development in carriers of RET mutations in exon 10.
\end{abstract}

Endocrine-Related Cancer (2009) 16 171-177

\section{Introduction}

Representing an excellent model of genotype-phenotype relationships in hereditary tumors, multiple endocrine neoplasia type 2A (MEN 2A) continues to arouse the keen interest of basic scientists and clinical researchers. Over the past 10-15 years, the molecular foundations of this heritable tumor syndrome of medullary thyroid cancer, pheochromocytoma, and parathyroid hyperplasia and adenoma have emerged: missense mutations - and exceptionally in-frame insertions and deletions - in the rearranged during transfection (RET) proto-oncogene in few 'hotspot' codons (Machens \& Dralle 2006). Located on chromosome 10q11.2, the RET gene codes for a transmembrane receptor tyrosine kinase in tissues of neural-crest derivation ('first hit'), giving rise to preneoplastic C-cell hyperplasia, adrenal medullary hyperplasia, and parathyroid chief cell hyperplasia. With the accrual of additional somatic mutations ('second hit'), these neuroendocrine cells undergo transformation to medullary thyroid cancer, pheochromocytoma, and parathyroid adenoma (Machens \& Dralle 2006).

Based on their position relative to the plasma membrane, MEN 2A-associated missense mutations in the RET tyrosine kinase receptor broadly fall into three categories, each of which is thought to have its unique mechanism of action: 
(i) mutations in the extracellular cysteine-rich domain through ligand-independent RET receptor dimerization and cross phosphorylation (codon 634>630>609, 611, 618, 620; high-risk category; Carlomagno et al. 1997, Ito et al. 1997, Chappuis-Flament et al. 1998);

(ii) mutations in the transmembrane domain presumably through non-covalent receptorreceptor interactions keeping RET monomers in close proximity to each other (codon 649; low-risk category; Kjær et al. 2006, ColomboBenkmann et al. 2008); and

(iii) mutations in the intracellular tyrosine kinase domain through facilitation of adenosine triphosphate binding by reorientation of critical amino acids (codons 768, 790, 791, 804, and 891; least-high risk category; Pasini et al. 1997, Plaza-Menacho et al. 2005).

Intriguingly, the in vitro transforming activity of RET mutations correlates with the age-related penetrance of medullary thyroid cancer, pheochromocytoma, and parathyroid tumors, and the penetrance of the full MEN 2A syndrome. Because thyroid C cells are more susceptible to constitutive receptor activation than adrenal medullary and parathyroid cells, medullary thyroid cancer usually develops earlier than pheochromocytoma and parathyroid disease (Machens \& Dralle 2006).

Six to 16 percent of RET families with extracellulardomain mutations in exon 10 (codons 609, 611, 618, and 620) develop a peculiar phenotype (Mulligan et al. 1994, Decker et al. 1998) consisting of:

(i) a gain-of-function MEN 2A phenotype through constitutive RET receptor activation; and

(ii) a loss-of-function Hirschsprung's disease (HSCR) phenotype through trapping of precursor RET proteins in the endoplasmatic reticulum, precluding their transport to the cell membrane. Decreased cell surface concentrations of RET protein are believed to cause the premature arrest of the craniocaudal migration of enteric neurons during the 5-12th week of gestation. The absence of autonomic ganglion cells within intestinal parasympathetic Meissner's and Auerbach's plexuses explains the HSCR symptoms of functional obstruction and megacolon (Carlomagno et al. 1996, Iwashita et al. 1996).

No loss-of-function HSCR phenotype has been reported in carriers of mutations in codons 630 and 634 of exon 11 (Mulligan et al. 1994, Decker et al. 1998). These codons are positioned very near the transmembrane domain of the RET receptor, which spans codons 636 to 657 (Kashuk et al. 2005, Kjær et al. 2006, Fig. 1). Functional studies demonstrated that the more N-terminal the mutation is from exon 11 , the more likely the mutated RET protein is to be trapped in the endoplasmatic reticulum, resulting in functional haploinsuffiency (Clowes et al. 2008).

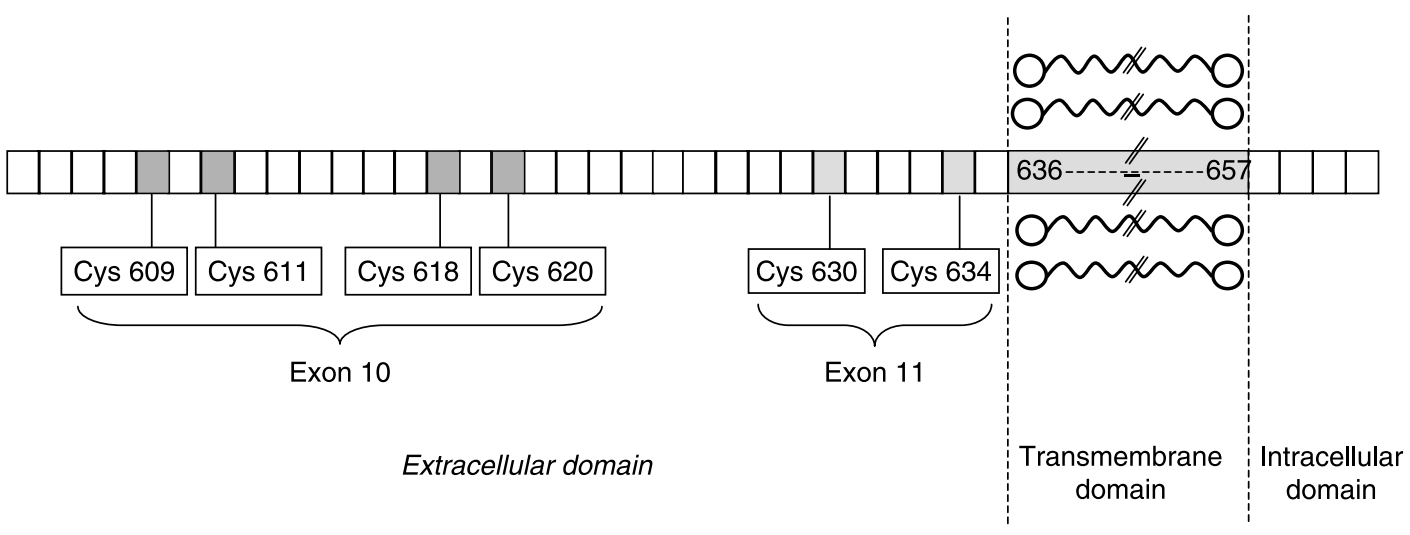

$\begin{array}{lccccccc}\text { Transforming } & 0.2 & 0.3 & 0.2 & 0.2 & 0.6 & 1 & 12 \text { days after transfection }{ }^{\dagger} \\ \text { activity in vitro } & 0.4 & - & 0.5 & 0.5 & 0.6 & 1 & 14 \text { days after transfection }{ }^{\ddagger}\end{array}$
(relative)

Figure 1 Schematic representation of cysteine residues in the extracellular domain of the rearranged during transfection (RET) tyrosine kinase receptor and resulting in vitro transforming activity. †: Ito et al. 1997, ‡: Chappuis-Flament et al. 1998. 
For carriers of mutations in exon 10 (codon 620, $618,611,609)$, these functional data may also have implications regarding the gain-of-function MEN 2A phenotype. This comparative study was undertaken to test the hypothesis that closer proximity of these mutations to the cell membrane translates into earlier or more advanced disease.

\section{Patients and methods}

\section{Patients}

Between November 1994 and May 2008, 295 RET gene carriers (90 index and 205 non-index patients) underwent thyroidectomy or cervical reoperation(s) at this institution. By the time they were having these operations, 194 RET carriers $(66 \%)$ had developed medullary thyroid cancer(s). Of the 194 carriers, 38 (20\%) harbored RET mutations in exon 10 (14 index and 24 non-index patients), 22 of whom had been studied previously in a different context (Machens et al. 2003b). To put these data into perspective, clinicopathologic results from these 38 carriers were compared with corresponding information from 80 carriers of mutations in exon 11 (codon 634, 78 patients; codon 630, two patients) operated on for medullary thyroid cancer during the same time period (25 index and 55 non-index patients).

\section{Genetic testing}

Before undergoing genetic testing, all patients or their legal guardians had given informed consent. For identification of RET germ-line mutations, genomic DNA was purified from peripheral blood leukocytes using standard techniques. Genomic DNA was amplified using PCR and oligonucleotide primers for exons $10,11,13,14,15$, and 16. Single-strand conformation polymorphism analysis and direct sequencing were performed according to national laboratory regulations for RET analysis.

\section{Extent of surgery}

All surgical procedures were conducted with optical magnification and bipolar coagulation, as described previously (Machens et al. 2002). Total thyroidectomy with systematic lymph node dissection in at least the central neck compartment had been performed in 37 out of the 38 carriers of RET mutations in exon 10 whereas one patient underwent total thyroidectomy without lymph node dissection. This 41-year-old carrier of a RET mutation in codon 611 revealed smaller than $1 \mathrm{~mm}$ medullary thyroid cancers confined to the thyroid gland. Because her calcitonin levels normalized after thyroidectomy and she had no evidence of recurrence 55 months postoperatively, she was considered node-negative. Six of the 38 patients also underwent adrenalectomy for pheochromocytoma at this institution or elsewhere (three patients each), as described previously (Machens et al. 2005). Informed consent was obtained for each surgical procedure, all of which represented standard practice of care.

\section{Pathological examination and staging}

After gross evaluation by the pathologist, the entire thyroid gland was divided vertically to separate the left and right lobes. The thyroid halves were then sectioned horizontally from the superior to the inferior pole, as described elsewhere (Hinze et al. 1998). After fixation in formalin, the whole thyroid gland was embedded in paraffin. Soft tissue and lymph nodes were processed separately. Conventional staining (hematoxylin and eosin) and calcitonin immunohistochemistry were performed on every surgical specimen, using the standard avidin-biotin complex peroxidase approach and a commercial polyclonal antibody (Immunotech, Marseilles, France). A diagnosis of medullary thyroid cancer was made on evidence of tumor extension beyond the basement membrane, demonstration of lymphatic or vascular invasion on histopathology, or a combination thereof. Primary tumor diameter was ascertained by direct measurements on the surgical specimens. When multiple medullary thyroid cancers were present, only the largest tumor was considered. Although a diagnosis of nodal metastasis usually required pathological confirmation, this need was waived for distant metastasis when there was such unequivocal evidence on ultrasonography, computed tomography, magnetic resonance imaging, positron emission tomography, or a combination thereof. As for medullary thyroid cancer, each diagnosis of pheochromocytoma was founded on histopathological analysis.

\section{Statistical analysis}

All demographic and histopathological data were stratified by mutated RET codon. Because the various amino acids substituting for cysteine were shown to confer similar RET transforming activity in vitro (Ito et al. 1997), no distinction was made based according to amino acid substitution. Categorical and continuous data were tested with the two-tailed Fisher exact test and one-way ANOVA respectively. The level of significance was set at $<0.05$. 


\section{Results}

Altogether, 38 RET carriers were identified with medullary thyroid cancer and germ-line mutations in codon 620 (8 patients from 8 families), 618 (19 patients from 8 families), 611 (10 patients from 2 families), and 609 (one patient from a single family). None of these RET carriers had a history of Hirschsprung's disease or developed parathyroid tumors (Table 1).

Exhibiting broadly comparable ages at the time of diagnosis (means of 33.4-39.3 years; $P=0.71$ ), the carriers of the various exon 10 mutations under comparison had been observed for an equal amount of time and thus been given equal opportunity to acquire those 'secondary hit' mutations required for tumor formation. The closer the mutations in codon 620,618 , and 611 were located to the transmembrane domain (codons 657-636) of the RET receptor, the greater were mean primary tumor diameters $(23.5$, 18.7 , and $7.5 \mathrm{~mm}, P=0.020)$ and the frequency of lymph node metastasis $(75,68$, and $30 \%, P=0.11)$ and pheochromocytoma $(38,16$, and $0 \%, P=0.11)$. When mutations in adjoining codons were collapsed (codons $620 / 618$ vs $611 / 609$ ), the differences in mean primary tumor diameter (20.1 vs $7.4 \mathrm{~mm}, P=0.005)$ and lymph node metastasis (70 vs $36 \% ; P=0.07$ ) were accentuated (Table 1). All other examined variables did not differ significantly among the various groups (Table 1).

By way of contrast, observational time differed tremendously between carriers of exon 10 and exon 11 mutations (Table 1). Because medullary, adrenal, and parathyroid disease in MEN type 2A are all time dependent, the carrier's age at the time of surgical intervention will greatly influence the histopathology of all tissues removed during the operation. Although they were almost 14 years younger (22.9 vs 36.8 years; $P<0.001$ ), carriers of exon 11 mutations had already grown medullary thyroid cancers two-thirds the size of cancers seen in carriers of exon 10 mutations (11.0 vs $16.5 \mathrm{~mm} ; P=0.04)$. Despite having been given much less opportunity to acquire somatic mutations, medullary thyroid cancers tended to be more often multifocal (77 vs $61 \% ; P=0.08$ ) among carriers of exon 11 mutations who more often displayed additional pheochromocytomas (33 vs $16 \% ; P=0.08$ ) and parathyroid hyperplasia or adenomas (10 vs $0 \%$; $P=0.05)$.

\section{Discussion}

Despite the limited number of RET carriers with medullary thyroid cancer, the present investigation demonstrated significant differences in primary tumor diameter of the largest medullary thyroid cancer among the carriers of exon 10 mutations following comparable periods of observation. Only equal time of observation enables direct comparisons of histopathologic data among the mutational groups. The histopathologic differences among the groups were proportional to the distance of the affected cysteine residues from the cell membrane, pointing to a doseeffect relationship. The frequency of lymph node metastases was more than twice as high in carriers of mutations in codon 620 or 618 , which are merely 16 and 18 amino acid residues away from the cell membrane, than in carriers of mutations in codon 611 or 609, which are 25 and 27 (i.e. 9 additional) amino acid residues away from it. Indeed, larger medullary thyroid cancers spread more often to regional lymph nodes than smaller ones (Machens et al. 2003a). Although all RET mutations in exon 10 can produce MEN 2A phenotypes (Machens et al. 2005), only carriers of mutations in codon 618 or 620 (but not codon 611 or 609) had pheochromocytomas. Remarkably, the former group comprised 2.5 times as many RET carriers (27 vs 11 carriers) from more than five times as many RET families (16 vs 3 families) than the latter. Commensurate with the much closer proximity of exon 11 mutations to the cell membrane (just 2-6 amino acid residues away from it, as opposed to 16-27 amino acid residues), carriers of exon 11 mutations required significantly fewer time to develop even more tumors than carriers of exon 10 mutations. These data buttress the concept of a relationship between cell membrane proximity and tumor development in MEN type 2A. By analogy, this relationship should extend to the various exon 10 mutations as well, among which existing differences in tumor development were more subtle.

Two modes of action, not mutually exclusive, may explain the observed differences among the 38 carriers of RET mutations in exon 10:

\section{Membrane proximity as an important determinant of disulfide bridge formation (explanation 1)}

In the native RET protein, C609/C611, and C618/C620 are believed to be disulfide linked, as are C630/C634. Any mutation resulting in the loss of one of these cysteines (e.g., C618) in one RET molecule would then set free the respective partner cysteine (e.g., C620) to form disulfide links with another RET molecule. The greater the distance of the liberated cysteine from the cell membrane, the lower the chance of getting close enough to another RET molecule to create the transforming disulfide bond. Indeed, mutations in 
Table 1 Multiple endocrine neoplasia (MEN) 2A-associated endocrine tumors grouped by rearranged during transfection (RET) germ-line mutations in exon 10

\begin{tabular}{|c|c|c|c|c|c|c|c|c|c|c|}
\hline & \multicolumn{4}{|c|}{ Affected exon 10 codons $^{a}$} & \multicolumn{3}{|c|}{ Categorized mutations within exon 10} & \multicolumn{3}{|c|}{ Exon 10 versus exon 11 mutations } \\
\hline & $\begin{array}{c}\text { C611 } \\
n=10 \\
\text { (2 families) }\end{array}$ & $\begin{array}{c}\text { C618 } \\
n=19 \\
\text { (8 families) }\end{array}$ & $\begin{array}{c}C 620 \\
n=8 \\
\text { (8 families) }\end{array}$ & $P^{b}$ & $\begin{array}{c}\text { C611/609 } \\
n=11 \\
\text { (3 families) }\end{array}$ & $\begin{array}{c}\text { C620/618 } \\
n=27 \\
\text { (16 families) }\end{array}$ & $P^{\mathrm{b}}$ & $\begin{array}{c}\text { C609 - C620 } \\
n=38 \\
\text { (19 families) }\end{array}$ & $\begin{array}{c}\text { C630' }- \text { C634 } \\
n=80 \\
\text { (59 families) }\end{array}$ & $P^{\mathrm{b}}$ \\
\hline \multicolumn{11}{|l|}{ Demographics } \\
\hline $\begin{array}{l}\text { Age at operation for } \\
\text { MTC }^{d} \text {, years, mean } \\
(95 \% \mathrm{Cl})\end{array}$ & $\begin{array}{c}39.3 \\
(33.0 ; 45.6)\end{array}$ & $\begin{array}{c}37.5 \\
(28.3 ; 46.7)\end{array}$ & $\begin{array}{c}33.4 \\
(25.4 ; 41.3)\end{array}$ & 0.71 & $\begin{array}{c}38.1 \\
(31.9 ; 44.3)\end{array}$ & $\begin{array}{c}36.3 \\
(29.6 ; 42.9)\end{array}$ & 0.74 & $\begin{array}{c}36.8 \\
(31.9 ; 41.7)\end{array}$ & $\begin{array}{c}22.9 \\
(19.3 ; 26.5)\end{array}$ & $<0.001$ \\
\hline Gender, male, $n(\%)$ & $2(20)$ & $9(47)$ & $2(25)$ & 0.32 & $3(27)$ & $11(41)$ & 0.49 & $14(37)$ & $35(44)$ & 0.55 \\
\hline Index patients, $n(\%)$ & $2(20)$ & $8(42)$ & $4(50)$ & 0.44 & $2(18)$ & $12(44)$ & 0.16 & $14(37)$ & $25(31)$ & 0.68 \\
\hline \multicolumn{11}{|l|}{ Medullary thyroid cancer ${ }^{d}$} \\
\hline $\begin{array}{l}\text { Primary tumor diameter, } \\
\mathrm{mm} \text {, mean }(95 \% \mathrm{Cl})^{\mathrm{e}}\end{array}$ & $\begin{array}{c}7.5 \\
(2.1 ; 12.9)\end{array}$ & $\begin{array}{c}18.7 \\
(12.2 ; 25.2)\end{array}$ & $\begin{array}{c}23.5 \\
(12.2 ; 34.8)\end{array}$ & 0.020 & $\begin{array}{c}7.4 \\
(2.7 ; 12.2)\end{array}$ & $\begin{array}{c}20.1 \\
(14.8 ; 25.4)\end{array}$ & 0.005 & $\begin{array}{c}16.5 \\
(12.1 ; 20.8)\end{array}$ & $\begin{array}{c}11.0 \\
(8.1 ; 14.0)\end{array}$ & 0.04 \\
\hline $\begin{array}{l}\text { Multifocal tumor growth, } \\
n(\%)\end{array}$ & $7(70)$ & $11(58)$ & $5(63)$ & 0.90 & $7(64)$ & $16(59)$ & $>0.99$ & $23(61)$ & $60(77)^{f}$ & 0.08 \\
\hline $\begin{array}{l}\text { Extrathyroidal extension, } \\
n(\%)\end{array}$ & $1(10)$ & $3(16)$ & $1(13)$ & $>0.99$ & $1(9)$ & $4(15)$ & $>0.99$ & $5(13)$ & $4(5)^{f}$ & 0.15 \\
\hline $\begin{array}{l}\text { Lymph node metastasis, } \\
n(\%)\end{array}$ & $3(30)$ & $13(68)$ & $6(75)$ & 0.11 & $4(36)$ & $19(70)$ & 0.07 & $23(61)$ & $34(43)^{g}$ & 0.11 \\
\hline $\begin{array}{l}\text { Distant metastasis at the } \\
\text { time of operation, } n(\%)\end{array}$ & 0 & $1(5)$ & $1(13)$ & 0.46 & 0 & $2(7)$ & $>0.99$ & $2(5)$ & $6(8)$ & $>0.99$ \\
\hline \multicolumn{11}{|c|}{$\begin{array}{l}\text { Pheochromocytoma, } \\
n(\%)\end{array}$} \\
\hline Unilateral & 0 & $3(16)$ & $3(38)$ & 0.11 & 0 & $6(22)$ & 0.15 & $6(16)$ & $26(33)$ & 0.08 \\
\hline Contralateral & 0 & $2(11)$ & $1(13)$ & 0.58 & 0 & $3(11)$ & 0.54 & $3(8)$ & $16(20)$ & 0.11 \\
\hline $\begin{array}{l}\text { Parathyroid hyper- } \\
\text { plasia/adenoma, } n(\%)\end{array}$ & 0 & 0 & 0 & $>0.99$ & 0 & 0 & $>0.99$ & 0 & $8(10)$ & 0.05 \\
\hline
\end{tabular}

RET, rearranged during transfection proto-oncogene; MTC, medullary thyroid cancer.

${ }^{a}$ Excluding information on the one and only MTC patient harboring a RET germ-line mutation in codon 609.

'Two-tailed Fisher's exact test and one-way ANOVA respectively.

${ }^{\mathrm{c}}$ Two non-index carriers.

$d_{100 \%}$ of patients (inclusion criterion)

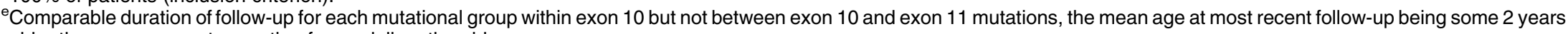
older than mean age at operation for medullary thyroid cancer.

fExcluding two carriers with missing information.

${ }^{g}$ Excluding one carrier with missing information. 
codons farther away from the cell membrane, such as C570W, W324C, C197Y, C157S/W/Y, C142S, Y96C, and R77C, generate solely HSCR phenotypes as they do not cause aberrant dimerization of RET receptor molecules (Kashuk et al. 2005, Kjær et al. 2006).

\section{Membrane proximity as an important determinant of RET protein transport to the cell membrane (explanation 2)}

In transfection experiments, transforming activity and expression of RET on the cell surface was comparable among mutations in codon $609,611,618$, or 620 twelve and fourteen days after transfection (Ito et al. 1997, Chappuis-Flament et al. 1998; Fig. 1), irrespective of the amino acid substitution for cysteine (Ito et al. 1997). This expression was three- and fivefold weaker than with mutations in codon 630 and 634, but similar to that one with HSCR-associated mutations P64L and F393L (Ito et al. 1997). It is unclear whether longer incubation of cell cultures would yield differences in RET transforming activity among mutations in codon 609, 611, 618, and 620 .

RET mutations associated with the long-segment HSCR phenotype were shown to impair the transport of RET proteins to the plasma membrane more severely than short-segment RET mutations (Iwashita et al. 1996). Most HSCR phenotypes result from mutations at multiple loci, most frequently coding mutations in the RET proto-oncogene acting in concert with second site non-complementing mutations (Kashuk et al. 2005). This may explain the absence of Hirschsprung's disease among our carriers of RET mutations in exon 10 and favor explanation 1.

More investigations are needed to dissect the individual contributions of either mode of action to the MEN 2A phenotype. With an estimated prevalence of only 9\% (13 of 141 RET families), germ-line mutations in exon 10 represent a rare condition, especially when they involve codon 609 or 611 (Machens \& Dralle 2008). This has rendered it difficult to accrue carriers of exon 10 RET mutations, which confer moderate transforming activity in vitro (Ito et al. 1997, Chappuis-Flament et al. 1998; Fig. 1), in sufficient numbers to demonstrate biological effects of moderate size. By implication, more patients are needed for moderate biologic effects to show than for strong biologic effects that attain statistical significance more easily.

Because of the worldwide proliferation of RET screening and widespread adoption of the concept of prophylactic thyroidectomy, the opportunity of observing the full spectrum of medullary thyroid cancer is fading away quickly. To raise statistical power, far longer follow-up periods over several decades will be required to increase the event rates for adrenal and parathyroid disease. Intriguing from a basic science perspective, the observed clinical differences among our patients with exon 10 mutations - contrasted with the disparities between carriers of exon 10 and 11 mutations - are too small to warrant differential treatment of carriers of RET mutations in codon $609,611,618$, and 620, all of which entail a high life-time risk of medullary thyroid cancer.

\section{Declaration of interest}

The authors declare that there is no conflict of interest that could be perceived as prejudicing the impartiality of the research reported.

\section{Funding}

This research did not receive any specific grant from any funding agency in the public, commercial or not-for-profit sector.

\section{Acknowledgements}

The authors are indebted to the physicians and institutions involved in the care of our patients for providing valuable information.

\section{References}

Carlomagno F, De Vita G, Berlingieri MT, De Franciscis V, Melillo RM, Colantuoni V, Kraus MH, Di Fiore PP, Fusco A \& Santoro M 1996 Molecular heterogeneity of RET loss of function in Hirschsprung's disease. EMBO Journal 15 2717-2725.

Carlomagno F, Salvatore G, Circafici AM, De Vita G, Melillo RM, De Franciscis V, Billaud M, Fusco A \& Santoro M 1997 The different RET-activating capability of mutations of cysteine 620 or cysteine 634 correlates with the multiple endocrine neoplasia type 2 disease phenotype. Cancer Research 57 391-395.

Chappuis-Flament S, Pasini A, De Vita G, Ségouffin-Cariou C, Fusco A, Attié T, Lenoir GM, Santoro M \& Billaud M 1998 Dual effect on the RET receptor of MEN 2 mutations affecting specific extracytoplasmic cysteines. Oncogene 17 2851-2861.

Clowes VE, Shaw-Smith C, Simpson H, Ball SG \& Acerini CL 2008 MEN2 screening dilemmas in a family with a novel RET mutation in the MEN2 susceptibility region of the gene, a family history of Hirschsprung's disease, and no family history of MEN2-related tumours. Clinical Endocrinology 68 666-670. 
Colombo-Benkmann M, Li Z, Riemann B, Hengst K, Herbst H, Keuser R, Groß U, Rondot S, Raue F, Senninger N et al. 2008 Characterization of the RET proto-oncogene transmembrane domain mutation S649L associated with nonaggressive medullary thyroid carcinoma. European Journal of Endocrinology 158 811-816.

Decker RA, Peacock ML \& Watson P 1998 Hirschsprung disease in MEN 2A: increased spectrum of RET exon 10 genotypes and strong genotype-phenotype correlation. Human Molecular Genetics 7 129-134.

Hinze R, Holzhausen HJ, Gimm O, Dralle H \& Rath FW 1998 Primary hereditary medullary thyroid carcinoma C-cell morphology and correlation with preoperative calcitonin levels. Virchows Archiv 433 203-208.

Ito S, Iwashita T, Asai N, Murakami H, Iwata Y, Sobue G \& Takahashi M 1997 Biological properties of RET with cysteine mutations correlate with multiple endocrine neoplasia type $2 \mathrm{~A}$, familial medullary thyroid carcinoma, and Hirschsprung's disease phenotype. Cancer Research 57 2870-2872.

Iwashita T, Murakami H, Asai N \& Takahashi M 1996 Mechanism of ret dysfunction by Hirschsprung mutations affecting its extracellular domain. Human Molecular Genetics 5 1577-1580.

Kashuk CS, Stone EA, Grice EA, Portnoy ME, Green ED, Sidow A, Chakravarti A \& McCallion AS 2005 Phenotype-genotype correlation in Hirschsprung disease is illuminated by comparative analysis of the RET protein sequence. PNAS 102 8949-8954.

Kjær S, Kurokawa K, Perrinjaquet M, Abrescia C \& Ibáñez CF 2006 Self-association of the transmembrane domain of RET underlies oncogenic activation by MEN2A mutations. Oncogene 25 7086-7095.

Machens A \& Dralle H 2006 Multiple endocrine neoplasia type 2 and the RET protooncogene: from bedside to bench to bedside. Molecular and Cellular Endocrinology 247 34-40.
Machens A \& Dralle H 2008 Familial prevalence and age of RET germline mutations: implications for screening. Clinical Endocrinology 69 81-87.

Machens A, Hinze R, Thomusch O \& Dralle H 2002 Pattern of nodal metastasis for primary and reoperative thyroid cancer. World Journal of Surgery 26 22-28.

Machens A, Holzhausen HJ, Lautenschläger C, Thanh PN \& Dralle H $2003 a$ Enhancement of lymph node metastasis and distant metastasis of thyroid carcinoma: a multivariate analysis of clinical risk factors. Cancer $\mathbf{9 8}$ 712-719.

Machens A, Holzhausen HJ, Thanh PN \& Dralle H $2003 b$ Malignant progression from $\mathrm{C}$-cell hyperplasia to medullary thyroid carcinoma in 167 carriers of RET germline mutations. Surgery 134 425-431.

Machens A, Brauckhoff M, Holzhausen HJ, Thanh PN, Lehnert H \& Dralle H 2005 Codon-specific development of pheochromocytoma in multiple endocrine neoplasia type 2. Journal of Clinical Endocrinology and Metabolism 90 3999-4003.

Mulligan LM, Eng C, Healey CS, Clayton D, Kwok JB, Gardner E, Ponder MA, Frilling A, Jackson CE, Lehnert $\mathrm{H}$ et al. 1994 Specific mutations of the RET protooncogene are related to disease phenotype in MEN2A and FMTC. Nature Genetics 6 70-74.

Pasini A, Genestre O, Legrand P, Schlumberger M, Rossel M, Fournier L, Rudkin BB, Schuffenecker I, Lenoir GM \& Billaud M 1997 Oncogenic activation of RET by two distinct FMTC mutations affecting the tyrosine kinase domain. Oncogene 15 393-402.

Plaza-Menacho IP, Koster R, Van der Sloot AM, Quax WJ, Osinga J, Van der Sluis T, Hollema H, Burzynski GM, Gimm O, Buys $\mathrm{CH}$ et al. 2005 RET-familial medullary thyroid carcinoma mutants Y791F and S891A activate a Src/JAK/STAT3 pathway, independent of glial cell line-derived neurotrophic factor. Cancer Research $\mathbf{6 5}$ 1729-1737. 